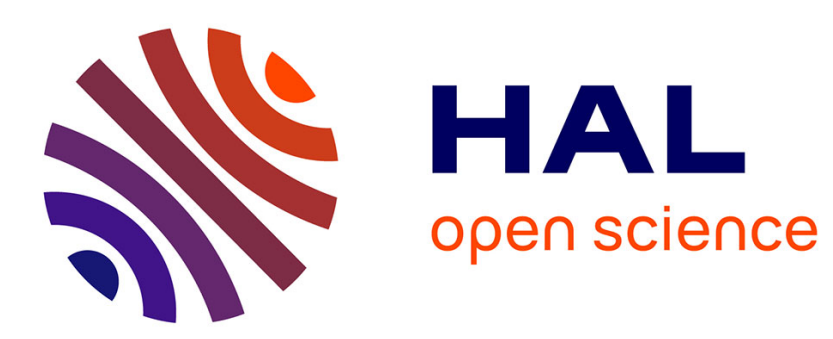

\title{
L'évaluation à travers les émotions : le cas d'estime et de déception
}

\author{
Iva Novakova, Julie Sorba
}

\section{To cite this version:}

Iva Novakova, Julie Sorba. L'évaluation à travers les émotions : le cas d'estime et de déception. Langue française, 2014, 184 (4), pp.75-91. 10.3917/lf.184.0075 . hal-01844358

\section{HAL Id: hal-01844358 \\ https://hal.science/hal-01844358}

Submitted on 9 Aug 2018

HAL is a multi-disciplinary open access archive for the deposit and dissemination of scientific research documents, whether they are published or not. The documents may come from teaching and research institutions in France or abroad, or from public or private research centers.
L'archive ouverte pluridisciplinaire HAL, est destinée au dépôt et à la diffusion de documents scientifiques de niveau recherche, publiés ou non, émanant des établissements d'enseignement et de recherche français ou étrangers, des laboratoires publics ou privés. 


\section{L'ÉVALUATION À TRAVERS LES ÉMOTIONS : LE CAS D'ESTIME ET DE DÉCEPTION}

Iva Novakova, Julie Sorba

Armand Colin | « Langue française »

2014/4 $\mathrm{n}^{\circ} 184 \mid$ pages 75 à 91

ISSN 0023-8368

ISBN 9782200929428

Article disponible en ligne à l'adresse :

https://www.cairn.info/revue-langue-francaise-2014-4-page-75.htm

\section{Pour citer cet article :}

Iva Novakova, Julie Sorba « L'évaluation à travers les émotions : le cas d'estime et de déception », Langue française 2014/4 (n²184), p. 75-91.

DOI 10.3917/lf.184.0075

Distribution électronique Cairn.info pour Armand Colin.

(C) Armand Colin. Tous droits réservés pour tous pays.

La reproduction ou représentation de cet article, notamment par photocopie, n'est autorisée que dans les limites des conditions générales d'utilisation du site ou, le cas échéant, des conditions générales de la licence souscrite par votre établissement. Toute autre reproduction ou représentation, en tout ou partie, sous quelque forme et de quelque manière que ce soit, est interdite sauf accord préalable et écrit de l'éditeur, en dehors des cas prévus par la législation en vigueur en France. Il est précisé que son stockage dans une base de données est également interdit. 


\section{Iva Novakova}

Université Grenoble-Alpes - Université Stendhal-Grenoble 3 \& Laboratoire LIDILEM (EA 609)

\section{Julie Sorba}

Université Grenoble-Alpes - Université Stendhal-Grenoble 3 \& Laboratoire LIDILEM (EA 609)

\section{L'évaluation à travers les émotions : le cas d'estime et de déception}

\section{INTRODUCTION}

D'après P. Charaudeau (2000 : 127), les émotions sont inséparables d'une interprétation s'appuyant sur des valeurs ou, plus précisément, sur un jugement d'ordre moral. Elles sont liées à « un savoir de croyances polarisé autour de valeurs socialement constituées » (op. cit. : 131). Les émotions impliquent à la fois la visée d'un objet (comme par exemple l'estime de X pour Y) ou d'une cause (la déception de $\mathrm{X}$ à cause de $\mathrm{Z}$ ), ainsi qu'un processus d'évaluation de l'objet ou de la cause (cf. Amossy 2006 ; Micheli 2010). D'ailleurs, l'axe plaisir/déplaisir est l'une des principales dimensions de la topique des émotions (Plantin, 1997 : 88). En outre, selon les théories de l'appraisal (Scherer 2001), les émotions, leur type et leur intensité sont déterminés par l'évaluation subjective, par le système de valeurs individuelles ou par celui des normes sociales ${ }^{1}$. Notre objectif ici est de proposer une analyse des marques linguistiques d'évaluation ou d'appréciation à travers l'étude des deux lexies d'émotion estime et déception. Nous nous demandons de quelle façon ces deux noms d'émotion contribuent à l'expression du jugement et, plus généralement, de l'évaluation au sein du discours journalistique.

Après la présentation de notre méthodologie et de nos corpus (section 2), nous étudions successivement, aux niveaux phrastique (section 3) et transphrastique (section 4), les profils discursifs des deux lexies afin d'établir les stratégies évaluatives mises en œuvre. Nous proposerons enfin une modélisation de notre 
analyse (section 5) pour mieux cerner les différents moyens d'expression de l'affectivité et de l'évaluation, ainsi que le lien entre ces dernières dans le discours.

\section{CHOIX DES LEXIES, MÉTHODOLOGIE, CORPUS}

Les noms estime et déception ont été choisis car ils renvoient à deux types d'affects différents ${ }^{2}$ : estime (l'estime de $\mathrm{X}$ envers/pour $\mathrm{Y}$ ) désigne un affect interpersonnel ayant un expérient $(\mathrm{X})$ et un objet $(\mathrm{Y})$, duratif, de polarité positive ; déception appartient à la classe des affects causés (la déception de $\mathrm{X}$ à cause de $\mathrm{Z}$ ), réactifs et ponctuels, de polarité négative. Nous utilisons le terme d'émotion comme une étiquette conventionnelle pour des lexies dénotant aussi bien l'émotion que le sentiment, y compris dans sa dimension sociale ${ }^{3}$.

Les différences sémantiques entre les deux noms permettent de les contraster sur le plan du jugement évaluatif auquel ils participent. Estime, comparé à déception, a une plus forte dimension sociale ou axiologique, inhérente à son sémantisme. Il s'agit d'un affect impliquant un jugement et une appréciation morale, moins présents, au premier abord, dans le sens de déception. Ainsi, les ressources lexicographiques (Petit Robert, TLFi) définissent estime comme un sentiment favorable à l'égard d'une personne et de ses qualités ou encore comme une appréciation positive de ces qualités qui méritent admiration et respect. Pour sa part, déception est une émotion davantage liée à une désillusion, à un désenchantement, à des attentes non satisfaites. L'agent causateur de la déception $(Z$ déçoit $X)$ provoque chez $X$ un ressenti désagréable et, de là, un jugement évaluatif négatif. Pourtant, si la dimension axiologique est inhérente à estime, elle est moins saillante dans le sens de déception, sans pour autant lui faire défaut. Le jugement portant sur l'objet de l'affect est d'emblée présent dans le sens d'estime ; dans celui de déception, il se réalise de façon moins directe, par le biais d'une évaluation négative portée sur la cause de l'émotion.

D'un point de vue méthodologique, l'étude des scénarios évaluatifs générés par les deux lexies s'appuie sur l'analyse systématique de leur combinatoire lexico-syntaxique ${ }^{4}$. À cela s'ajoute une analyse fondée sur des méthodes lexico-statistiques (Diwersy 2012 ; Blumenthal 2012 ; Diwersy \& Kraif 2013) permettant de repérer, sur de grands corpus, les accompagnateurs préférentiels (co-occurrents) des lexies. Au niveau phrastique d'abord, nous examinons les associations lexicales et les configurations actancielles spécifiques d'estime et

2. L'antonyme d'estime, à savoir mépris, n'a pas été retenu car il appartient au même type d'affect interpersonnel qu'estime et a donc un fonctionnement plus ou moins identique dans la construction de l'évaluation, ce qui n'est pas le cas d'un affect causé comme déception qui a des caractéristiques différentes.

3. Pour la distinction entre émotions, sentiments et états affectifs, établie dans un objectif classificatoire des noms d'affect, cf. Goossens (2005), Tutin et al. (2006).

4. À propos des différents paramètres de la combinatoire lexico-syntaxique qui nous renseignent sur les spécificités des lexies des émotions, cf. Tutin et al. (2006), Novakova \& Tutin (2009). 
de déception. Au niveau de la macrostructure textuelle ensuite, nous explorons, d'une part, les réseaux isotopiques tissés autour des deux lexies, et, d'autre part, les sous-genres textuels (ou rubriques journalistiques) dans lesquelles chacune d'elles apparaît de manière privilégiée (étude des topiques ${ }^{5}$ spécifiques). Nous observons donc de quelle façon s'organise et se construit l'évaluation dans le discours journalistique.

Notre corpus, constitué dans le cadre du projet Emolex (www.emolex.eu) ${ }^{6}$, regroupe deux années de parution (2007-2008) de quatre quotidiens français (Le Monde, Libération, Le Figaro, Ouest-France) pour un total de 100 millions de mots. Nous avons extrait de façon automatique, grâce à l'interface EmoConc ${ }^{7}$, toutes les occurrences des deux termes, estime (877) et déception (2 055). Après une phase manuelle de nettoyage des occurrences non pertinentes (comme par exemple succès d'estime qui ne renvoie pas à l'acception 'émotion'), sont retenus tous les accompagnateurs de la lexie d'émotion qui s'avèrent préférentiels sur la base du calcul probabiliste $\log$-likelihood ${ }^{8}$ (Blumenthal 2012). Ensuite, les associations sémantiques spécifiques choisies sont codées selon des dimensionsvaleurs sémantiques (voir section 3.1). Lorsque les échantillons ne sont pas équilibrés, les calculs statistiques effectués sont fondés sur l'indice de spécificité log-likelihood normalisé, afin de minimiser le biais introduit par ces différences de fréquence (Diwersy, 2012 : 91) ${ }^{9}$. Les résultats décrits au niveau phrastique correspondent à ces fréquences. Au niveau textuel, nous avons analysé les 200 premières séquences de chacune des lexies : il s'agit d'un échantillon représentatif pour l'analyse des isotopies, mais aussi des rubriques dans lesquelles les deux lexies apparaissent de manière récurrente. Les séquences textuelles sont composées d'un empan de 3 à 5 lignes incluant les métadonnées (titre, auteur, date), mais la totalité du paragraphe d'où elles sont extraites demeure accessible.

\section{L'ANALYSE DES LEXIES AU NIVEAU PHRASTIQUE}

À ce niveau, sont analysées d'abord les associations lexicales dans lesquelles apparaissent estime et déception, puis leur mise en série aux côtés d'autres lexies d'émotion. Ces paramètres, constitutifs du profil lexical d'estime et de déception,

5. Sur l'organisation des topiques au sein des médias d'information, en particulier la télévision, cf. Charaudeau (2000 : 141). L'auteur définit les topiques comme étant des imaginaires socio-discursifs.

6. Projet franco-allemand ANR/DFG (ANR-09-FASHS-017), coordonné par I. Novakova (U. Stendhal) et P. Blumenthal (U. de Cologne).

7. La base de données, créée à l'issue du projet Emolex, est consultable en accès libre (http://emolex.ugrenoble3.fr/emoBase/). Les outils de traitement des corpus Emolex permettent d'extraire de manière automatique les accompagnateurs des mots qui s'avèrent spécifiques sur la base de leur lemme.

8. Le seuil de spécificité a été fixé à 10,83.

9. Pour le calcul du log-likelihood normalisé, l'outil créé par S. Diwersy (U. de Cologne) dans le cadre du projet Emolex fonctionne sous le logiciel R et est implémenté dans le module EmoLing de la base de données EmoBase (http://emolex.u-grenoble3.fr/emoLing/index.php). 
sont révélateurs des différentes facettes de l'évaluation construite autour de ces deux noms. S'y ajoute l'étude de leurs schémas actanciels et de leurs positions récurrentes au sein de la phrase, permettant d'établir leur profil syntaxique.

\subsection{Les associations lexicales spécifiques}

Il s'agit ici d'analyser les associations lexicales entre le mot pivot (estime ou déception) et ses collocatifs ${ }^{10}$ (témoignage d'estime, énorme déception) selon une grille de dimensions-valeurs sémantiques. Celles-ci sont établies à partir des traits sémantiques des collocatifs avec lesquels les mots pivots entrent en cooccurrence. Voici un extrait de cette grille ${ }^{11}$ :

Tableau 1 : Grille des dimensions-valeurs sémantiques

\begin{tabular}{|l|l|l|}
\hline Dimensions & Valeurs & Exemples \\
\hline \multirow{2}{*}{ Intensité } & forte & très grande estime ; immense déception \\
\cline { 2 - 3 } & faible & peu d'estime ; petite déception \\
\hline \multirow{2}{*}{ Polarité } & positive & jouir de l'estime de qn \\
\cline { 2 - 3 } & négative & manque d'estime \\
\hline \multirow{2}{*}{ Manifestation } & physique & afficher sa déception ; marque d'estime \\
\cline { 2 - 3 } & externe & mesurer la déception de qn \\
\hline Verbalisation & émotif/communicatif & dire son estime ; avouer sa déception \\
\hline \multirow{2}{*}{ Aspect } & phasique & $\begin{array}{l}\text { gagner, conserver, perdre l'estime de qn ; surmon- } \\
\text { ter sa déception }\end{array}$ \\
\hline Contrôle & émotion/manifestation & dissimuler sa déception \\
\hline
\end{tabular}

L'observation du corpus montre que les deux noms ont, dans des proportions variables, des collocations spécifiques pour les dimensions intensité, polarité, manifestation, verbalisation. Déception présente aussi des collocatifs exprimant le contrôle de l'émotion, ce qui n'est pas le cas d'estime. Inversement, estime s'associe souvent avec des collocatifs aspectuels. Il est donc possible de distinguer les profils lexicaux des deux mots à travers leurs associations lexicales statistiquement spécifiques.

\subsubsection{La polarité et l'intensité}

La polarité avec ses deux valeurs - positive et négative - est une dimension sémantique résolument axiologique (cf. Grutschus, Kern \& Tutin 2013). Le plus souvent, l'emploi d'estime dans le corpus journalistique est lié à une appréciation positive en adéquation avec les normes sociales ou morales. Il s'agit alors d'un jugement généralement produit par l'expérient $(X)$ à l'égard de l'objet de l'affect (Y), par exemple $Y$ \{bénéficie de, mérite, jouit de, est digne de\} l'estime de $X$ :

10. Nous utilisons ici le terme de « collocation » au sens de l'association entre une base (ou pivot), comme examen, célibataire, colère) et un collocatif (passer, endurci, bouffée de) (Blumenthal \& Hausmann, 2006 : 4).

11. Cette grille a été élaborée dans le cadre du projet Emolex à partir de Goossens (2005) et de Tutin et al. (2006). 
Ces héros oubliés non seulement méritent toute notre estime et notre reconnaissance, mais ils redorent à eux seuls l'image bien ternie d'un " mammouth » ingérable qui ne sait résoudre ses problèmes qu'en exigeant toujours davantage de moyens. Rendons hommage à tous ces fidèles serviteurs de la République pour lesquels le mot « sacerdoce » a encore un sens. (Le Figaro, 16.01.2008)

Dans l'exemple (1), issu de la rubrique «Courrier » des lecteurs, l'évaluation est centrée sur l'objet de l'estime : ces enseignants « héros », exerçant leur métier avec passion et dévouement, auxquels le lecteur (l'expérient $X$ ) rend hommage.

Par ailleurs, la présence de collocatifs verbaux de polarité négative comme perdre l'estime ou manquer d'estime fait basculer estime dans une axiologie plutôt négative ${ }^{12}$. On peut, par son comportement ou ses actes, perdre l'estime de ses proches ou du public :

(2) En choisissant de collaborer avec le FBI (Z), il (Y) a perdu l'estime de sa femme (X), et surtout, de ses deux enfants. (Le Monde, 06.06.2008)

Le jugement négatif de l'expérient (sa femme, ses enfants) est confirmé en (2) par l'expression de la cause (la collaboration avec le FBI) mise en apposition et en position frontale dans l'énoncé. Évaluation et sentiment se trouvent alors profondément imbriqués (cf. aussi Charaudeau 1992). Une divergence de polarité est également observée lorsque la lexie se combine avec des collocatifs exprimant l'intensité faible (sa faible estime, avoir peu d'estime pour qn, tenir qn en modeste estime). Le peu d'estime est une façon atténuée d'exprimer la mésestime, la mauvaise opinion de l'expérient envers l'objet du ressenti, et donc une évaluation dépréciative, comme par exemple en (3) :

(3) Plusieurs sondages ont démontré que les Marocains ont peu d'estime pour l'action du Parlement actuel. (Le Figaro, 06.09.2007)

Par ailleurs, estime apparaît surtout dans deux collocations statistiquement spécifiques pour l'intensité forte en convergence avec la polarité positive : tenir en haute estime et avoir beaucoup d'estime pour qn. Déception offre, quant à elle, une plus grande variété de collocatifs intensifs qui mettent en relief la polarité négative inhérente de cet affect : \{(très) grosse, grande, immense, profonde, énorme, réelle, forte, terrible, cruelle, sévère $\}$ déception.

Déception s'associe également de manière privilégiée avec l'adjectif axiologique légitime. Ainsi, la collocation déception légitime relève de la " polarité axiologique » (Grutschus, Kern \& Tutin 2013). L'évaluation, dans ce cas, vient de l'extérieur ; autrement dit, on approuve, on justifie qu'un tel ressent de la déception (4): 
(4) C'est un coup d'arrêt, on voulait plus que tout gagner ce match, la déception de Guillaume Comméat était légitime, on a manqué d'efficacité comme souvent. (Ouest France, 04.02.2008)

En bref, l'étude fine de la combinatoire montre que certains accompagnateurs peuvent faire basculer estime vers une polarité négative (ex. 2-3). En revanche, déception ne change pas de polarité, mais celle-ci peut être intensifiée ou qualifiée comme étant un affect « justifié, correct, tel qu'il doit être » (Grutschus, Kern \& Tutin, 2013 : 87). Les données confirment l'importance des paramètres de la polarité et de l'intensité, souvent imbriquées, dans la construction de l'évaluation autour des affects ${ }^{13}$. Pour estime, l'évaluation porte sur l'objet (Y) de l'affect ; pour déception, l'affect est négatif, désagréable pour l'expérient (X). Il est provoqué par une cause sur laquelle on porte implicitement un jugement négatif.

\subsubsection{Manifestation et verbalisation}

Les dimensions manifestation et verbalisation sont également liées à l'évaluation construite autour des deux émotions. L'estime comme la déception se manifestent, se montrent et, aussi souvent, se disent: \{marques, déclarations, témoignages\} d'estime; \{afficher, avouer\} sa déception; \{déclarer, dire, redire\} son estime pour qn. En effet, comme les collocatifs pour la manifestation et la verbalisation expriment l'extériorisation de l'affect ressenti, il s'agit là d'un acte volontaire de jugement exprimé par l'expérient, qui va le plus souvent de l'intérieur vers l'extérieur :

(5) Lors du débat de confiance au Parlement, le président du Conseil avait multiplié gestes de paix et marques d'estime envers le leader de l'opposition. (Le Figaro, 17.05.2008)

(6) Mais, à plusieurs proches, il a confié sa « déception » sur une campagne qu'il juge pour le moment médiocre et pas à la hauteur des enjeux pour la France. (Libération, 12.03.2007)

En (5), le président du Conseil tient à montrer son estime envers le leader de l'opposition en en multipliant les marques. En (6), la campagne jugée « médiocre » provoque un sentiment de déception chez l'expérient, sentiment qu'il choisit d'exprimer à ses proches. L'analyse par dimensions sémantiques permet aussi de contraster les deux lexies dans le fonctionnement de l'évaluation. Ainsi, comme nous allons le voir, le pivot déception attire des accompagnateurs spécifiques pour la dimension contrôle, tandis qu'estime se combine avec des collocatifs exprimant l'aspect.

13. Sur les notions de polarité en lien avec l'intensité, en tant que propriétés caractéristiques des énoncés évaluatifs, cf. aussi Zhang \& Ferrari (2014, ce volume). 


\subsubsection{Le contrôle}

Cette dimension regroupe des collocatifs qui renvoient à la capacité de l'expérient à contrôler l'affect qu'il éprouve ou ses manifestations. Elle est caractéristique et fréquente pour déception, probablement à cause de la polarité négative de la lexie. On s'efforce de surmonter, d'oublier, d'évacuer, de digérer sa déception, comme en (7), où le patineur passe rapidement outre sa troisième place dévalorisante aux Championnats d'Europe :

(7) «J'ai très vite digéré ma déception en Croatie. » (Le Figaro, 21.03.2008)

Souvent, la déception échappe au contrôle (avoir du mal \{à cacher, à dissimuler, à retenir\} sa déception, ne pas cacher sa déception). Ces collocations sont très fréquentes dans les rubriques sportives. En (8), le dirigeant du club porte un jugement ouvertement négatif vis-à-vis de la mauvaise performance de ses joueurs :

Bien évidemment Jean-Pierre Pinon, le dirigeant rennais ne cachait pas sa déception : "Quand on mène 4-1, on a le match grandement en main, on n'a pas le droit de faire match nul ». (Ouest France, 05.03.2007)

L'impossibilité de dissimuler le sentiment de déception est un indice révélateur d'une évaluation négative ; le groupe adverbial de sens appréciatif bien évidemment placé en tête de phrase ${ }^{14}$ confirme la légitimité de l'affect.

L'estime, quant à elle, renvoie à un ressenti positif qui s'inscrit dans la durée, que l'on tend à afficher, à montrer, que l'on témoigne plus volontiers à quelqu'un et que l'on n'a pas besoin de contrôler. Aucune collocation pour la dimension contrôle de l'affect n'a été relevée dans le corpus pour estime lorsqu'il a sa polarité positive.

\subsubsection{L'aspect}

La dimension aspectuelle est surtout présente dans les associations lexicales autour d'estime $\left(18,5 \%\right.$ contre 1,1\% pour déception $\left.{ }^{15}\right)$. L'objet de l'affect gagne, regagne, remonte, regrimpe dans l'estime des gens; il conserve, restaure, retrouve l'estime des autres ou de soi. Ces associations lexicales montrent que cet affect de nature axiologique peut s'inscrire dans la durée (9) ou traverser différentes phases (10) :

(9) Nous n'avons pas la même vision de la fin, mais, notez-le, je conserve la plus grande estime pour lui. (Libération, 03.05.2007)

(10) Un score si important signifie que les Français ont souhaité renouer avec des valeurs d'estime et de respect, qui avaient été abandonnées. (Libération, 07.05.2007)

14. Sur le rôle des adverbes dans le processus d'évaluation et de jugement, cf. Jackiewicz (2014, ce volume).

15. Il s'agit surtout de la collocation d'aspect ponctuel inchoatif la déception pointe. 
L'estime et le respect sont considérés comme des valeurs positives que l'on doit préserver dans le temps, ce qui n'est pas le cas de la déception, un affect déplaisant, plus ponctuel que l'on a tendance à évacuer.

\subsection{Les lexies en série}

L'emploi d'estime et de déception en combinaison avec d'autres noms d'émotion est un élément caractéristique de leur profil lexical qui fournit des indices sur le lien entre émotion et évaluation. En effet, les lexies, conformément à leurs propriétés sémantiques inhérentes, ont des préférences lorsqu'elles s'associent avec d'autres noms d'émotion. Estime a tendance à former des séries avec des noms qui expriment des émotions (considération, respect, admiration, affection, reconnaissance), mais aussi des attitudes ou des valeurs morales (confiance, fidélité, soutien, fierté), ce qui renforce sa dimension évaluative :

(11) Les joueurs sont applaudis dans la rue, signent des autographes. «On les a vus les épaules se redresser à mesure qu'ils retrouvaient le respect, la confiance et l'estime de soi qu'ils avaient perdus ». (Libération, 30.07.2007)

Déception, quant à elle, forme tendanciellement beaucoup moins de séries qu'estime. Il s'agit plutôt d'un affect nommé à l'état pur ${ }^{16}$. Lorsque le mot se combine avec d'autres noms d'émotions, celles-ci expriment souvent la conséquence de la déception : déception et colère, déception et exaspération, déceptions et frustrations, déception et incompréhension, amertume et déception :

Certains électeurs socialistes expriment, ou exprimaient, en effet, déception, frustration, voire dépit, vis-à-vis de la personnalité ou de la campagne de Ségolène Royal. (Ouest France, 23.02.2007)

Les lexies frustration et dépit attestées dans l'entourage de déception en (12), mais aussi d'autres noms comme espoir, attentes ou échec ailleurs, révèlent des éléments du scénario prototypique de la lexie (attentes non satisfaites, promesses non tenues, résultats médiocres).

\subsection{Le profil syntaxique}

Pour établir le profil syntaxique des lexies, nous étudions les configurations actancielles qu'elles réalisent, ainsi que les positions qu'elles préfèrent ou évitent dans la phrase (ou « colligations » selon Hoey 2005).

16. Déception est, à cet égard, comparable à un autre affect causé comme stupeur (pour plus de détails, cf. Novakova \& Sorba 2014). 


\subsubsection{Les configurations actancielles}

Les deux lexies sont prototypiquement bivalentes (réalisation de deux actants ${ }^{17}$ ). L'analyse des données montre qu'estime apparaît le plus souvent dans des emplois où sont réalisés l'expérient $(X)$ et $l^{\prime}$ objet $(Y){ }^{18}$ sur lequel porte le jugement positif :

(13) Nous $(\mathrm{X})$ sommes nombreux ici à afficher pour le général de Gaulle $(\mathrm{Y})$ une admiration et une estime immenses. (Le Figaro, 21.05.2008)

Néanmoins, les structures actancielles des noms peuvent présenter de nombreuses variations, suite aux visées discursives du locuteur. En effet, le locuteur peut choisir de centrer ${ }^{19}$ son évaluation sur la cause (Z) de l'estime (ou de l'absence d'estime) en éliminant de la structure phrastique l'actant X (l'expérient) :

Pour ce qu'il fut et ce qu'il fit ( $\mathrm{Z}$ ), le colonel Kadhafi (Y) ne mérite que peu d'estime. (Le Monde, 15.12.2007)

En (14), la cause $(Z)$ est mise en relief grâce à sa position frontale dans l'énoncé. Y figure aussi l'objet de l'estime (Y), le colonel Kadhafi, tandis que l'expérient $(X)$ est effacé. Le locuteur fait le choix de ne pas le nommer pour donner à sa formulation un caractère absolu (le colonel Kadhafi ne mérite pas d'être estimé). Il existe aussi des cas où une fusion actancielle est observée :

$\mathrm{J}^{\prime}(=\mathrm{X})$ ai de l'estime et même de l'admiration pour son parcours (Z/Y) donc en tant que personne c'est quelqu'un qui m'intéresse. (Libération, 16.10.2007)

En (15), ce sont la cause ( $\mathrm{Z}$ ) et $\mathrm{l}^{\prime}$ objet $(\mathrm{Y})$ qui ont fusionné (l'estime de $\mathrm{X}$ envers $\mathrm{Y}$ à cause de $\mathrm{Z}=$ son parcours). La fusion actancielle correspond à une stratégie discursive plus économique que la configuration actancielle complète à trois actants sémantiques (Asé), rare mais attestée :

(16) Car, [...] Bertrand Poirot-Delpech (Y), par sa gentillesse et par son talent (Z), a acquis l'estime et l'affection de tous (X). (Le Monde, 09.12.2008)

Sont réalisés en (16), l'objet de l'estime ( $\mathrm{Y}: \mathrm{B}$. Poirot-Delpech), la cause ( $\mathrm{Z}$ : par sa gentillesse et son talent) et, enfin, $\mathrm{l}^{\prime}$ expérient $(\mathrm{X}:$ de tous). La réalisation complète des actants confère à l'énoncé et, de là, à l'évaluation toute sa complétude discursive. Les schémas actanciels variés réalisés autour d'estime révèlent le caractère multiforme de l'évaluation (cf. Eensoo \& Valette 2014, ce volume). Si l'expérient, comme on l'a vu, peut ne pas être réalisé dans l'énoncé, l'objet de

17. Sur la méthodologie d'identification des actants syntaxiques (Asy) et sémantiques (Asé) et sur les interactions entre les profils discursifs et les structures actancielles des noms surprise et respect, cf. Novakova, Grossmann \& Goosens (2013).

18. Notre codage des actants s'inspire de Mel'čuk et al. (1984/1992), mais notre approche, à la différence de la Théorie Sens-Texte, reste entièrement surfaciste.

19. Pour la notion de « centrage discursif », cf. Fesenmeier (2010). 
l'estime, lui, est toujours présent car il constitue ainsi la cible évaluée, sur laquelle est porté le jugement.

En général, déception réalise des schémas actanciels plus dépouillés qu'estime : il privilégie les emplois avalents (sans actant), comme en (17), ou monovalents (un seul actant, l'expérient, réalisé sous forme de pronom possessif), comme en (18) :

\section{On constate une grande déception. (Le Figaro, 24.02.2007)}

Mais la branche néerlandaise de Greenpeace ne cache pas sa déception. (Libération, 19.02.2007)

Dans la configuration avalente (17), l'affect n'est pas attribué à un expérientévaluateur clairement identifié au niveau phrastique. On pourrait toutefois le retrouver dans un contexte plus large. La lexie d'affect peut être aussi considérée comme un embrayeur qui amorce la présentation des différents actants en jeu dans l'évaluation :

(19) Seule déception : on a beau fouiller les pages indiquées par l'entrée Fellini de l'index, on ne saura jamais comment Vidal atterrit à Rome. (Libération, 17.03.2007)

De plus, même si le second Asé cause peut ne pas être exprimé, il n'est jamais très loin dans la phrase. Il apparaît après deux points (19), dans le dispositif du discours direct (20) ou dans des citations entre guillemets (21) :

(20) Simba Makoni ne cache pas sa déception : «L'espérance de vie, qui était de 74 ans, a été divisée en deux ». (Le Figaro, 29.03.2008)

(21) Déception d'Alain Perrin : « C'est sûr qu'il y a une grosse déception parce que c'est un objectif qui s'en va ». (Le Figaro, 17.01.2008)

Ces procédés de mise en scène de la cause $Z$ de la déception ont une incidence sur l'évaluation, en l'occurrence, négative de cette dernière. Le journaliste porte une évaluation indirecte, en prenant de la distance. Il insère la voix d'énonciateurs seconds dans des séquences de discours direct rapporté (20-21). Ce procédé vient alors renforcer le jugement négatif formulé par l'évaluateur. On pourrait parler ici d'un effet polyphonique de l'évaluation, absent dans le cas d'estime.

\subsubsection{Les positions récurrentes}

Les deux lexies n'occupent pas les mêmes positions syntaxiques dans la phrase. Selon la théorie du Lexical Priming de M. Hoey (2005), l'emploi d'un mot ou d'une séquence de mots est lié à des associations sémantiques (semantic associations) préactivées (ou amorcées). L'auteur considère aussi que les items lexicaux sont activés en fonction de leur «préférence » ou de leur « aversion » pour certaines fonctions ou environnements grammaticaux (ce qu'il appelle des « colligations », Hoey, $2005: 44)$. Ainsi, déception privilégie nettement la position sujet (22), les constructions impersonnelles (23), mais aussi les titres et les phrases averbales, où la lexie apparaît en position frontale sans déterminant (24) : 
(23) «Il y a de la déception", admet, un brin embarrassé Chatel. (Le Figaro, 25.06.2008)

(24) Déception pour Peugeot, devancé par Audi, vainqueur pour la huitième fois en neuf éditions. (Le Figaro, 16.06.2008)

Ces positions et fonctions pourraient s'expliquer par le fait que souvent l'évaluateur (celui qui éprouve la déception) ainsi que la cause évaluée ne sont pas exprimés. L'émotion sur laquelle le journaliste-locuteur insiste est seulement " nommée », elle participe moins directement au dispositif discursif d'évaluation.

Estime, pour sa part, évite ces distributions et montre une nette préférence pour la fonction COD, ce qui lui permet de réaliser son schéma prototypique bivalent :

(25) Nombreux sont ceux qui lui témoignent leur estime. (Le Monde, 02.02.2007)

À la différence de déception, les actants d'estime (l'expérient-évaluateur et l'objet évalué) participent directement au dispositif de l'évaluation : son caractère est plus saillant, plus prévisible. Les actants sont présents et identifiables dans le cotexte. La prise en compte des colligations est donc un paramètre pertinent pour l'analyse du profil syntaxique des mots d'émotion et corollairement, des scenarios du jugement exprimé à travers eux.

\section{L'ANALYSE DES LEXIES AU NIVEAU TRANSPHRASTIQUE}

Notre objectif est ici de comprendre comment la posture évaluative est tissée dans le texte au niveau transphrastique. En effet, la subjectivité est conçue comme un phénomène continu, notamment avec nos unités lexicales marquées de façon relativement stable d'un trait de valorisation (estime) ou de dévalorisation (déception). Pour élaborer le profil textuel de chacune des lexies, nous étudions, dans un premier temps, les réseaux isotopiques ${ }^{20}$ développés autour de celles-ci dans nos séquences, avant d'examiner les sous-genres textuels (ou rubriques journalistique) dans lesquels apparaissent de manière privilégiée estime et déception.

\subsection{Les réseaux isotopiques}

Dans notre corpus, les isotopies de la polarité et de l'intensité sont particulièrement saillantes et récurrentes, ce qui concorde avec les observations réalisées au niveau phrastique (cf. 3.1.1). 


\subsubsection{Isotopie de la polarité}

Le sémantisme de la lexie estime offrant une polarité positive, nombreuses sont les séquences où cette isotopie est actualisée (ex. 1). De manière symétrique mais inversée, il en va de même pour déception (26) :

(26) «Le pire adversaire de l'Usap, c'est l'Usap... » Cette réflexion désabusée d'un dirigeant perpignanais en dit long sur la déception née de l'élimination en quart de finale de la Coupe d'Europe face aux London Irish. (Le Figaro, 07.04.2008)

Dans les exemples (1) et (26), on note la richesse et l'abondance des réseaux isotopiques de la polarité positive (héros, reconnaissance, redorent, hommage, fidèles, sacerdoce) ou négative (pire, désabusée, élimination).

Néanmoins, dans notre corpus journalistique, on observe un phénomène saillant avec la présence d'estime au sein d'un environnement textuel tissant une polarité négative ( $25 \%$ des occurrences) :

(27) Hormis Londres, nulle part ailleurs que dans ce nord-est de l'Angleterre la situation n'est plus calamiteuse [...]. « Un travail de longue haleine, qui suppose d'abord de gagner la confiance de ces exclus du système, reconnaît le conseiller Nick Cott. La bataille se mène dès la naissance, en aidant des parents en rupture de ban à assumer leur rôle ; ensuite à la maternelle, à travers l'éveil au langage. Car derrière la pauvreté infantile, il y a pire : le manque d'estime de soi, l'absence d'ambition qui condamnent beaucoup de ces jeunes au même échec que leurs parents. » (Libération, 27.06.2007)

Ce cotexte s'observe majoritairement (mais pas exclusivement) en compagnie de la collocation très figée estime de soi. Il est ainsi lié à l'évaluation négative de l'objet (cf. 3.1.1). Cette schématisation textuelle de polarité visiblement divergente entre pivot et cotexte ne se rencontre, pour déception, que de manière très sporadique. Cette tendance nettement observée pour estime peut sans doute s'expliquer par la topique ancrée dans son sémantisme : l'estime désigne un affect positif (notamment dans sa version estime de soi) qui, depuis les années 90, fait l'objet d'une promotion notamment dans les milieux éducatifs (Famose \& Bertsch, 2009 : 1).

\subsubsection{Isotopie de I'intensité}

La seconde isotopie notable tissée dans notre corpus autour des deux lexies est celle de l'intensité. Déception semble un affect davantage associé à une forte intensité (20\% des occurrences) qu'estime ( $8 \%$ ). Il nous semble que cette observation du corpus traduit au niveau textuel un phénomène observé au niveau syntagmatique, à savoir la plus grande variété des collocatifs d'intensité associé à déception. En (28), les éléments de l'isotopie de l'intensité développent les causes de l'affect (monceau, accumulées, plus grande) et ses conséquences (hargne, avec force) :

(28) Elles témoignent du monceau d'aigreurs accumulées - depuis l'esclavage jusqu'à la « Françafrique »-, et révélées par le discours de M. Sarkozy. [...]. Les 
promesses de M. Sarkozy leur avaient fait espérer une plus grande vigilance démocratique de la part de Paris. La profondeur de la déception est à la hauteur des attentes qui, même sous la hargne, s'expriment avec force. (Le Monde, 29.02.2008)

Cette tendance plus forte pour déception à apparaître dans cet environnement textuel est à l'image de la place de cet affect évaluatif dans notre société moderne fortement « déceptive » (Lipovetsky, 2006 : 18).

L'analyse de ces réseaux isotopiques saillants confirme le caractère textogène des affects qui engendrent dans leur sillage des chaînes anaphoriques riches et des scénarios détaillés (cf. Novakova \& Sorba 2013). Les isotopies relevées permettent de catégoriser l'énoncé comme évaluatif : le sémantisme des lexies génère une configuration textuelle où l'évaluation se propage de la lexie à son entourage (cf. Plantin, 2011 : 75).

\subsection{Les rubriques}

L'étude des rubriques ${ }^{21}$ ou sous-genres textuels dans lesquels apparaissent nos lexies est un moyen d'aborder la dimension culturelle des discours évaluatifs : exprimer un affect évaluatif, c'est se positionner par rapport à un système de normes et de valeurs. Le Tableau 2 présente la répartition des lexies estime et déception dans les différentes rubriques journalistiques :

Tableau 2 : Répartition des lexies dans les rubriques journalistiques

\begin{tabular}{|l|c|c|}
\hline & estime & déception \\
\hline Politique & $39 \%$ & $40 \%$ \\
\hline Culture & $21 \%$ & $8 \%$ \\
\hline Société & $29 \%$ & $15 \%$ \\
\hline Économie & $1 \%$ & $11 \%$ \\
\hline Justice & $3 \%$ & $1 \%$ \\
\hline Sport & $4 \%$ & $24 \%$ \\
\hline autres & $3 \%$ & $1 \%$ \\
\hline
\end{tabular}

Tout d'abord, estime et déception ont en commun d'apparaître, dans des proportions similaires $( \pm 40 \%)$ et de manière privilégiée, dans la rubrique Politique. C'est probablement un effet de corpus car la presse quotidienne réputée sérieuse constituant notre corpus n'est pas le lieu de l'étalage de la sphère intime : les déceptions rapportées relèvent presque exclusivement de la sphère publique (29) et il en va de même pour les témoignages d'estime (30) :

21. La rubrique est comprise ici comme un outil de «classification et de hiérarchisation de l'information » (Herman \& Lugrin, 1999 : 72). Dans notre classement, un article de presse relève d'une seule rubrique. La catégorie à laquelle appartient chaque rubrique se définit par « l'intention dominante et manifeste (affichée), c'est-à-dire telle que les lecteurs doivent la comprendre »(Grosse, 2001 : 51). 
Même au parti socialiste, elle régresse de $9 \%$. C'est donc un mouvement de déception assez largement répandu. Seules les prochaines semaines diront si ce revers est un simple trou d'air ou le début d'un éventuel dévissage. (Le Figaro, 03.02.2007)

(30) Pourtant, Hillary Clinton a l'air compétente, sérieuse, expérimentée... Et elle l'est ! Au Sénat, elle a gagné l'estime générale par son travail, sa connaissance des dossiers. (Le Monde, 20.04.2008)

Néanmoins, le monde moderne a développé des facteurs secrétant structurellement de la déception vis-à-vis du politique (cf. Lipovetsky, 2006 : 59-61), ce qui peut aussi expliquer la fréquence élevée de la lexie dans ce type de rubrique alors culturellement formatée pour l'expression de cet affect.

Par ailleurs, les occurrences d'estime se concentrent dans les rubriques Politique, Société, Culture, alors qu'une diversité plus prononcée se constate pour celles où apparaît déception : en effet, celle-ci se trouve également de manière non négligeable dans les rubriques Sport $(4,7,8)$ et Économie, alors que c'est un phénomène très marginal pour estime. Ainsi, déception apparaît de manière plus régulière qu'estime dans des rubriques plus diversifiées. Ce phénomène propre à déception est à relier à la multiplicité des lieux où la déception est susceptible de se produire ${ }^{22}$, tandis que pour estime, les situations culturellement formatées pour l'expression de cet affect sont moins diversifiées.

\section{MODÉLISATION ET CONCLUSION}

Notre objectif était de proposer une analyse des marques linguistiques d'évaluation à travers l'étude des lexies d'émotion estime et déception. Pour ce faire, nous avons élaboré le profil discursif de chacune d'entre elles afin de mieux cerner les différents moyens d'expression de l'affectivité et de l'évaluation ainsi que leur lien dans le discours. Nous présentons dans le Tableau 3 une modélisation de notre analyse fonctionnelle discursive construite à l'épreuve des faits :

Tableau 3 : Modélisation de l'analyse fonctionnelle discursive

\begin{tabular}{|c|c|c|}
\hline \multicolumn{2}{|c|}{ Profil discursif } \\
\hline \multicolumn{2}{|c|}{$\downarrow$} & $\downarrow$ \\
\hline$\downarrow$ & $\downarrow$ & Niveau transphrastique \\
\hline Niveau phrastique & $\downarrow$ \\
\hline$\downarrow$ & Profil lexical & $\downarrow$ \\
\hline configurations & $\downarrow$ & réseaux isotopiques \\
$\&$ & associations & $\&$ \\
colligations & $\&$ & sous-genres \\
\hline
\end{tabular}

22. Cf. Lipovetsky (2006 : 20) qui parle de «l'extension du domaine de la déception » dans notre société contemporaine (de la déception sentimentale à la déception politique, culturelle, consommatrice, etc.). 
L'analyse du profil discursif des lexies permet de contraster les dispositifs évaluatifs qu'elles génèrent. Au niveau phrastique, estime, émotion interpersonnelle positive, apparaît surtout dans des collocations présentant des variations de polarité (une grande estime/un manque d'estime), ce qui débouche sur une évaluation centrée sur l'objet-cible. Elle apparaît plus souvent en série, notamment en compagnie d'autres noms d'émotion, d'attitude ou de valeurs morales. Elle s'entoure davantage de ses actants (deux ou trois) et a une préférence pour la fonction de COD. Ses configurations actancielles variées témoignent du caractère multiforme de l'évaluation. Au niveau transphrastique, estime apparaît au sein d'un dispositif particulier (réseau isotopiques de la polarité négative) qui met en valeur, par un contraste fort, la dimension axiologique plus saillante dans le sémantisme de la lexie désignant alors un affect à promouvoir.

Pour sa part, déception, en tant qu'émotion négative causée, s'associe souvent à des collocatifs exprimant le contrôle de l'émotion ou de sa manifestation. Il est vrai que l'attente sociale implique un contrôle accru de la verbalisation des émotions négatives. En outre, on trouve moins de collocatifs pour la dimension polarité que dans le cas d'estime, mais ceux-ci s'orientent davantage vers une polarité axiologique (déception légitime) centrant l'évaluation négative sur la cause qui provoque l'affect. De plus, déception entre moins souvent dans des séries, présente des structures actancielles nettement moins développées, voire absentes, et a une préférence pour les positions frontales, les titres et les phrases averbales, où elle est simplement nommée. La lexie apparaît ainsi dans des constructions plus dépouillées qu'estime, ce qui permet de gommer la présence trop directe d'un évaluateur dans l'énoncé tout en le maintenant de manière diffuse. $\mathrm{Au}$ niveau transphrastique, la présence régulière de déception dans un plus large éventail de rubriques traduit, outre un certain air du temps, la plus grande potentialité narrative de cette émotion.

Ainsi, il ressort de notre étude que la «densité informationnelle» (Blumenthal, 2014 : 186) est plus grande pour estime que pour déception, même si l'on peut toujours trouver, pour chacune des deux lexies, une expression de la cause de l'affect évaluatif, à savoir un paramètre extérieur déclenchant une évaluation positive pour estime ou négative pour déception. Enfin, la dimension plus axiologique d'estime se confirme, tandis que la lexie déception, même si cette dimension est moins évidente dans son sémantisme de prime abord, participe néanmoins aux dispositifs évaluatifs mis en place dans l'énoncé.

\section{Références bibliographiques}

[PETIT RoBert] Le Petit Robert de la langue française. Dictionnaire alphabétique et analogique de la langue française. Nouvelle édition, 2014 (version 4.0).

[TLFI] Trésor de la langue française informatisé [http://atilf.atilf.fr/]

Amossy R. (2006), L'argumentation dans le discours, Paris : Armand Colin. 
Blumenthal P. (2012), "Méthodes statistiques en lexicologie contrastive ", in L. Begioni \& C. Bracquenier (éds), Sémantique et lexicologie des langues d'Europe, Rennes : Presses Universitaires de Rennes, 114-128.

Blumenthal P. (2014), "Caractéristiques et effets de la complexité sémantique de noms d'affect ", in P. Blumenthal, I. Novakova \& D. Siepmann (éds), Les émotions dans le discours / Emotions in discourse, Franckfurt am Main : Peter Lang, 175-186.

Blumenthal P. \& Hausmann F. J. (2006), "Présentation : collocation, corpus, dictionnaires ", Langue française 150, 3-13.

Charaudeau P. (1992), Grammaire du sens et de l'expression, Paris : Hachette.

Charaudeau P. (2000), "Une problématisation discursive de l'émotion. À propos des effets de pathémisation à la télévision ", in C. Plantin, M. Doury \& V. Traverso (éds), Les émotions dans les interactions, Lyon: Presses Universitaires de Lyon, 125-154.

DIWERSY S. (2012), Kookkurrenz, Kontrast, Profil. Korpusinduzierte Studien zur lexikalischsyntaktischen Kombinatorik französischer Substantive (mit ergänzenden Betrachtungen zum Deutschen), Berlin : de Gruyter.

DIWERSY S. \& KRAIF O. (2013), "Observations statistiques de cooccurrents lexico-syntaxiques pour la catégorisation sémantique d'un champ lexical ", in F. Baider \& G. Cislaru (éds), Cartographie des émotions. Propositions linguistiques et sociolinguistiques, Paris : Presses Sorbonne Nouvelle, 55-69.

EENSOO E. \& VALETTE M. (2014), "Approche textuelle pour le traitement automatique du discours évaluatif ", Langue française 184. (ce volume)

FAmose J.-P. \& BertSCH J. (2009), L'estime de soi : une controverse éducative, Paris : Presses Universitaires de France.

FESENMEIER L. (2010), "Se souvenir en français et en italien : différence(s) de centrage ", in M. Iliescu, H. Siller-Runggaldier \& P. Danler (éds), Actes du XXVe Congrès International de Linguistique et de Philologie Romanes, Tübingen : Max Niemeyer, 85-96.

GoosSENS V. (2005), "Les noms de sentiment : esquisse de typologie sémantique fondée sur les collocations verbales ", Lidil 32, 103-121.

GROSSE E.-U. (2001), "Évolution et typologie des genres journalistiques. Essai d'une vue d'ensemble ", Semen 13. [http://semen.revues.org/2615; consulté le 03.05.2014].

Grutschus A., KeRn B \& TUtin A. (2013), "La polarité du lexique de l'affect : perspective combinatoire et contrastive ", in F. Baider \& G. Cislaru (éds), Cartographie des émotions. Propositions linguistiques et sociologiques, Paris : Presses Sorbonne Nouvelle, 85-96.

HeRman T. \& LUgrin G. (1999), "La hiérarchie des rubriques : un outil de description de la presse ", Communication et langage 122, 72-85.

HOEY M. (2005), Lexical priming. A New Theory of Words and Language, Londres/New York: Routledge.

JACKIEWICZ A. (2014), "Apprécier des attitudes et des manières d'agir ", Langue française 184. (ce volume)

LIPOVETSKY G. (2006), La société de déception. Entretien mené par B. Richard, Paris : Éditions Textuel.

MEL'ČUK I. et al. (1984/1992), Dictionnaire explicatif et combinatoire du français contemporain. Recherches lexico-sémantiques, T. I-IV, Montréal : Presses de I'Université de Montréal.

Mıchel R. (2010), L'émotion argumentée. L'abolition de la peine de mort dans le débat parlementaire français, Paris : Cerf. 
Novakova I. \& SoRBA J. (2013), "Stupéfier et jalouser dans les séquences textuelles journalistiques : quel profil discursif pour quelle stratégie argumentative ? ", Le discours et la langue 4 (1), 203-220.

Novakova I. \& SoRBA J. (2014), "L'émotion dans le discours. À la recherche du profil discursif de stupeur et de jalousie ", in P. Blumenthal, I. Novakova \& D. Siepmann (éds), Les émotions dans le discours / Emotions in Discourse, Franckfurt am Main : Peter Lang, 161-175.

Novakova I. \& Tutin A. (éds) (2009), Le lexique des émotions, Grenoble : ELLUG.

Novakova I., Grossmann F. \& Goossens V. (2013), "Interactions entre profil discursif et structures actancielles : l'exemple des noms de surprise et de respect ", in F. Baider \& G. Cislaru (éds), Cartographie des émotions. Propositions linguistiques et sociolinguistiques, Paris : Presses Sorbonne Nouvelle, 71-83.

Plantin C. (1997), "L'argumentation dans l'émotion ", Pratiques 96, 81-100.

Plantin C. (2011), Les bonnes raisons des émotions. Principes et méthode pour l'étude du discours "émotionné ", Berne : Peter Lang.

RASTIER F. (1987), Sémantique interprétative, Paris : Presses Universitaires de France.

SCHERER K. R. (2001), “Appraisal considered as a process of multi-level sequential checking”, in K. R. Scherer, A. Schorr \& T. Johnstone (eds), Appraisal processes in emotion: Theory, Methods, Research, New York/Oxford: Oxford University Press, 92-120.

TUtin A. et al. (2006), "Esquisse de typologie des noms d'affect à partir de leurs propriétés combinatoires ", Langue française 150, 32-49.

ZHANG L. \& FERRARI S. (2014), "Intensité et polarité : un modèle opératoire articulant plusieurs travaux linguistiques", Langue française 184. (ce volume) 